\title{
A Novel WLAN Roaming Decision and Selection Scheme for Mobile Data Offloading
}

\author{
Nam Nguyen, Mohammad Arifuzzaman, and Takuro Sato \\ Graduate School of Global Information and Telecommunication Studies, Waseda University, Building No. 29-7, \\ 1-3-10 Nishi-Waseda, Shinjuku-ku, Tokyo 169-0051, Japan \\ Correspondence should be addressed to Nam Nguyen; namnh@moegi.waseda.jp
}

Received 31 July 2015; Revised 14 November 2015; Accepted 24 November 2015

Academic Editor: Francisco Martínez

Copyright (C) 2015 Nam Nguyen et al. This is an open access article distributed under the Creative Commons Attribution License, which permits unrestricted use, distribution, and reproduction in any medium, provided the original work is properly cited.

The existing IEEE and 3GPP standards have laid the foundation for integrating cellular and WiFi network to deliver a seamless experience for the end-users when roaming across multiple access networks. However, in recent studies, the issue of making roaming decision and intelligently selecting the most preferable Point of Service to optimize network resource and improve end user's experience has not been considered properly. In this paper, we propose a novel cellular and WiFi roaming decision and AP selection scheme based on state of the art, 3GPP TS24.312 and IEEE 802.11u, k standards. Our proposed scheme assists the mobile nodes to decide the right timing to make roaming decision and select preferable point of service based on the operator's policies and real-time network condition. We also introduce our simulation model of a heterogeneous network with cellular and WiFi interworking as well as 3GPP ANDSF, TS24.312. It is a complete end-to-end system model from application to physical layer with considering user's mobility and realistic traffic model. The proposed scheme outperformed the conventional WiFi selection scheme in terms of dynamically steering mobile node's data traffic from macrocell to available Access Points. The proposed scheme increased the utilization and balanced the traffic load of access points and improved user's experienced throughput.

\section{Introduction}

In recent years, the proliferation of smart devices (smart phones and tablets) and mobile Internet applications is generating huge demand for mobile data traffic. In addition, we are shifting from voice-centric homogeneous network toward data-oriented heterogeneous network (HetNet). It is predicted that mobile data traffic will grow more than 1000 times in next decades, and billions of devices will be connected due to the popularity of Internet of Things by then [1] Conversely, the licensed frequency band of cellular network is expensive and limited. Therefore, increasing the number of macrocell base stations is no longer an efficient method to boost network capacity. Therefore, offloading data traffic from the cellular network using alternative access network is becoming a major concern for network operators in order to avoid network congestion and degrading end-user's quality of experience (QoE). As the amount of data transfer increases, offloading is becoming increasingly important. The obvious choices for offloading are small cells and WiFi. The first option for data offloading is to deploy small-cells beneath the macrocell's coverage to increase the capacity as well as downlink and uplink throughput. However, small-cell technologies such as femtocell or picocell raise macrocell interference and cost-effective issues. On the other hand, WiFi is emerging as an effective alternative since it utilizes unlicensed frequencies, which causes less interference to macrocell. In addition, it is also very cost-effective compared to small-cell technology. For these reasons, more and more network operators are in favor of adopting WiFi on a large scale as the extended access network for their cellular network. WiFi will become increasingly important, playing the role of the third RAN, and will be the most reliable data offload technology in nextgeneration wireless network.

One of major challenges in WiFi offloading is to provide a smooth and seamless experience for end-user when roaming from cellular to WiFi APs and vice versa [2]. Most of researches in the literature are focused on this area [3-7]. 
However, there is another issue concerning how the mobile node decides the right timing to make roaming decision and selects the most preferable point of service anytime and anywhere. This issue becomes important to the operators deploying carrier WiFi AP for cellular offloading because their main concern is how to increase the utilization of available access points and proactively prevent network congestion for the macrocell. For example, currently, there are network operators such as AT\&T US, KDDI Japan, deploying WiFi APs in crowded area in order to offload traffic and prevent congestion for cellular network. However, since the mobile users are usually not aware of the availability of WiFi $\mathrm{AP}$, it is common that many of them might not switch their connection from cellular network to $\mathrm{WiFi}$ or the mobile terminals unconsciously connect to overloaded APs. As a result, the WiFi APs are typically underutilized and the end-users might experience bad QoS due to overload or congestion.

3GPP and IEEE have been actively working on the integration and interworking issues between 3GPP and non3GPP wireless networks. For instance, 3GPP released a new network architecture that is a new all-IP core network architecture known as Evolved Packet Core (EPC) with a new entity called Access Network Discovery and Selection Function (ANDSF) TS $24.312[8,9]$ Mobile IP, Proxy IP as well as IEEE 802.11u [10] or Hotspot 2.0 (HS2.0) [11], and so forth. These new standards are the key enablers to make roaming between 3GPP and non-3GPP access network as smooth and seamless as it is in 3GPP cellular network. However, as far as we are concerned, the issue of how to decide the right timing to make roaming decision and select preferable point of service has not been addressed adequately by the existing standards. Since the key standards have not been finalized and optimized for dynamic data offloading purpose, the new amendments are expected in future releases of TS24.312 [9] and IEEE 802.11u [10]. For instance, in 3GPP ANDSF TS 24.312, the main function of ANDSF host entity is to provide policies or rules for discovering and selecting preferable access network or IP routing policies in case of multiple homing User Entity (UE). However, these policies are generally predefined and unchanged. If UE sole uses ANDSF's policies and does not consider any real-time network condition or measured information from UE, it can lead to undesirable network selection issues for UE [12]. For example, if the AP candidate is temporary overloaded or far away, it will degrade end-user's experienced QoS. On the other hand, IEEE 802.11u standard was mainly targeted on providing a seamless transition experience from WiFi to cellular network. Although it provides a standardized mean to let UE obtain additional AP and backhaul network condition information such as the channel load, AP's downlink/uplink capability for enhancing AP discovery and selection, it does not specify how the UE should use this information piece to find and select the preferable point of service.

In this research, we address the aforementioned issue by proposing a novel cellular-WiFi roaming decision and selection scheme specified for mobile data offloading purpose. Our proposed scheme takes advantage of state-of-the-art 3GPP (24.312 ANDSF) and IEEE (IEEE 802.11u, k) standards. By combining ANDSF's policies and network condition metrics measured by UE, the UE autonomously decides the right timing to make cellular-WiFi roaming decision and selects the most preferable point of access. In addition, we also present our simulation model of 3GPP ANDSF and vertical handoff, which is a system level end-to-end model from physical layer to application layer. The model features typical HetNet scenario with vertical handover between WiMAX base stations and WiFi APs, the ANDSF host, and the connection manager running on each mobile node. We also take into account user's mobility and realistic traffic model. During the simulation process, the ANDSF server and the UE's connection manager entity behave and interact with other nodes corresponding to real-time network events. For evaluation, we compare the performance of proposed scheme with that of the conventional WiFi selection (based on the RSS/SINR adopted in most of popular smart devices). The simulation result shows that our proposed scheme can proactively steer the UE's traffic from macrobase stations to available WiFi APs, which improves both network resource utilization and UE's experienced throughput.

\section{Background and Related Work}

2.1. 3GPP Access Network Discovery and Selection Function. 3GPP has considered the non-3GPP access interworking issue for cellular network since 3GPP release 8 [8]. 3GPP proposed a tight-coupling architecture that integrates non3GPP access into 3GPP Evolved Packet Core (EPC). In addition, there is a new entity called Access Network Discovery and Selection Function (ANDSF) TS24.312 [9] appended to the EPC architecture. Its role is to provide the UEs with a set of rules for selecting preferable access network as well as IP routing policies for multihoming UE. Upon being provisioned with ANDSF's policies, the UE regularly checks the validity of the policies and selects the active rule with the highest priority. Validity criteria include date, time of day, or location. The UE can also use the valid routing policy (e.g., preferred RAT, specific preferable AP) to decide whether to route the ongoing traffic over $3 \mathrm{GPP}$ or WiFi. Researches on the ANDSF's functions and specification are ongoing and new enhancements and extensions for ANDSF's function are expected in future 3GPP releases.

2.2. IEEE 802.11u Standard. IEEE ratified an amendment for IEEE 802.11 standard to support WiFi and 3GPP network interworking known as IEEE802.11u [10]. This amendment was aimed at making roaming between WiFi and external network such as 3GPP network as smooth and seamless as roaming within 3 GPP cellular network. Based on this amendment, WiFi Alliance also released specification for the next generation of WiFi AP also known as Hotspot 2.0 (HS2.0) [11] or WiFi Certified Passpoint. Beyond the security authentication enhancement, the main feature of HS2.0 in release 1 was to facilitate the WiFi AP discovery and selection procedures of supported UEs. Therefore, the HS2.0 provides UE various type of network condition information (AP, backhaul load condition, authentication type, connection capacity, etc.) prior to association through the Access Network Query Protocol (ANQP), and the Generic Advertisement Service 
(GAS). These additional network condition metrics are beneficial to UE when selecting preferable AP as well as providing seamless handoff experience.

2.3. Related Works. In EPC architecture, the ANDSF is designated to deliver network selection policies from the operator for individual UE $[8,9]$. Therefore, the operators can impose the policies to control when, where, and which access network that the UE can connect. However, one of the most important aspects of dynamic network selection is to consider the real-time network conditions as the criteria for selection. The conventional WiFi offloading strategy (adopted by most of smart device) is that WiFi always has higher priority than $3 \mathrm{G} / 4 \mathrm{G}$ cellular. As a result, the UE switches to WiFi whenever AP is detected. Besides, WiFi AP selection is simply based on the Receiving Signal Strength (RSS) or SINR; thus the nearest AP is selected. However, in the dense area where there are large numbers of APs, we believe that RSS or SINR information alone is not enough to make intelligent $\mathrm{WiFi}$ selection. In such scenario, the selection scheme that is solely based on RSS can lead to bad quality of experience (QoE) for UE or network resource underutilization issue. Therefore, we need to take into account other factors to enhance roaming and point of service selection decision.

In [13] authors highlighted technical challenges in heterogeneous wireless networks underlying seamless vertical handover. The authors also presented a detailed survey on the vertical mobility management process with a focus on decision-making mechanisms. Handoff decision based on multiple inputs such as bandwidth, QoS, cost, and UE's velocity have been considered for years with complex system model and algorithms such as analytic hierarchy process (AHP), grey relational analysis (GRA) $[14,15]$. However, the authors failed to describe how to implement their proposal in reality because some required inputs such as available bandwidth, cost of the service, UE's velocity, connection jitter, and packet loss are not available or difficult to obtain with the existing standards.

In [12] 4GAmericas provided a comprehensive view of the state of the art of the key enablers for integrating cellular network and WiFi. It explained the possibility of combining the advantages of ANDSF and HS2.0 could resolve the problems of macrocell and WiFi roaming. However, there was no detail description, numerical evaluation such as simulations or experiment's result provided. However, there was no detail description, numerical evaluation such as simulations or experiment's result provided. In [16], authors examined the mobility between different access technologies in heterogeneous wireless networks and focused on the case of interoperability issue. The quality of the service of mobility, the time required for the handover and the packet loss during handover, and so forth are also extensively analysed. There was 3GPP task group working on WLAN and 3GPP radio interworking at radio level. In [17], the authors tried to improve access network selection and traffic steering decision between 3GPP LTE network and WLAN. In their proposal, the cellular network provided additional Received Channel Power Indicator (RCPI) and/or (Received Signal to Noise Indicator) RSNI thresholds to the terminal so that it could make roaming decision to WiFi when the LTE network condition was not favourable. In [18], the authors addressed solutions for WiFi offloading in LTE cellular networks when demands exceeded the capability of the LTE access. For evaluation, the authors compared the performance of each access technology using different network performance metrics. In [19], the authors proposed a novel networkassisted user-centric WiFi offloading model for maximizing per-user throughput in a heterogeneous network. In the proposed model, the network collects network information, such as the number of users in WiFi network and their traffic load. Subsequently, the network decided the specific portion of traffic to be transmitted via WiFi network so that the individual user's throughput could be maximized by offloading more traffic to WiFi. Through analysis, the authors investigated the effect of the WiFi offloading ratio on the per-user throughput. However, they did not consider user's mobility in their simulation model. In [20], the authors studied how much economic benefits can be anticipated thanks to delayed WiFi offloading method, by modeling the interaction between a single provider and users based on a two-stage sequential game. In this work, they first analytically proved that WiFi offloading is economically beneficial for both the provider and users. Their major focus was to understand how and how much users and the provider obtained the economic incentives by adopting delayed WiFi offloading and investigated the effect of different pricing and delaytolerance. In [21], the authors investigated the performance improvement induced by adopting a hybrid cellular/WiFi communication architecture where the mobile users can be served by either the LTE eNodeB or a mobile WiFi AP. In this proposed scheme, the mobile WiFi APs are considered as relay entities that are wirelessly connected to the LTE eNodeB and share this broadband connection with other users over WiFi tethering or ad hoc network (using WiFi frequencies). Important performance metrics of the proposed hybrid scheme including the average bit error probability (ABEP), capacity, and outage probability were theoretically studied.

The IEEE 802.21 in [22], Media-Independent Handover $(\mathrm{MIH})$, is a standardized framework proposed by IEEE, which facilitates vertical handover across heterogeneous networks. It defines a generic media-independent handoff (MIH) framework to support information exchange between network elements as well as a set of functional components to execute the roaming process. IEEE 802.21 specifies three media-independent services. Media-Independent Event Service (MIES), Media Independent Command Service (MICS), and Media-Independent Information Service (MIIS). The MIIS provides a data store of available networks and network parameters and defines standard query/response messages to access and retrieve such information for each available access network. The MIIS's role is similar to ANDSF. References $[23,24]$ provided an overview of vertical handoff approaches based on MIH framework. The work presented comprehensive solutions to ensure VHO between three types of different RATs: WiFi, WiMAX, and 3G, dealing with multiple parameters to make VHO decision. To the best of our knowledge, in recent years, researches related to MIH are gradually fading out from the academic studies and there is 


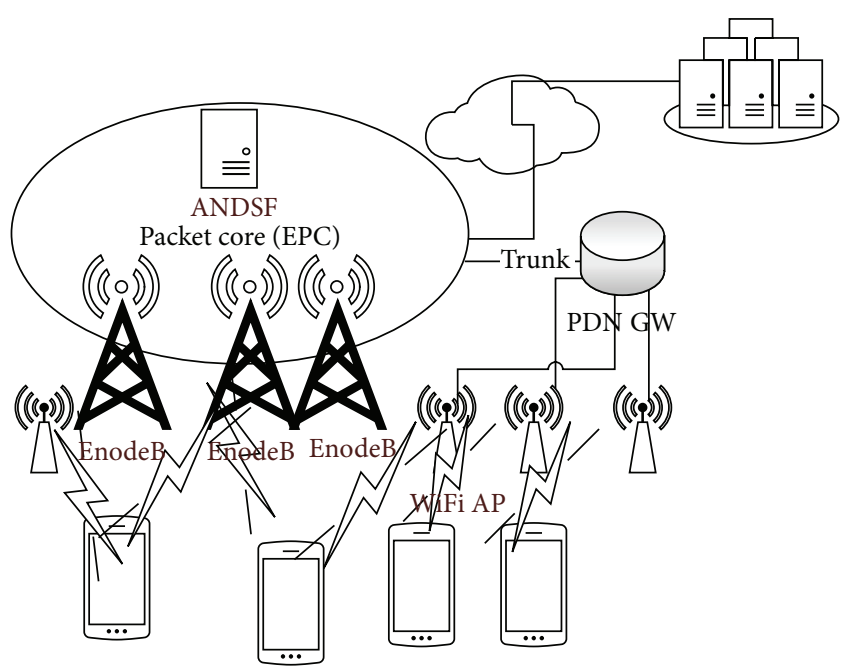

FIGURE 1: Heterogeneous network scenario with LTE cellular and WiFi interworking.

no initiative from industries to implement this standard in commercial products. The reason is that it required many changes and modifications to the MAC layer of existing IEEE 802.11, 802.16, 802.3 standards as well as 3GPP LTE, overall core network architecture, and protocols. In addition, the main function, MIIS service for network discovery, can be replaceable by ANDSF, which is more robust and popular. For these reasons, we consider using ANDSF instead of MIH to provide discovery and selection policy utilized in our scheme.

\section{The Proposed Cellular and WiFi Roaming and Selection Scheme}

3.1. The Proposed Scheme Formation Initiative. Our assumption is that we have a 3 GPP network operator providing highspeed mobile data service using cellular (e.g., LTE) and WiFi networks. It also extends to cellular carriers and third party $\mathrm{WiFi}$ providers as long as they have interworking agreement and interconnected network. This is typical HetNet scenario in future wireless network because we anticipate that providing high-speed data service via multiple access networks will become popular in near future. Figure 1 shows the baseline scenario when users move from LTE network to WiFi network coverage. Depending on preference, in this situation, the UEs may decide to switch their access interface from LTE to $\mathrm{WiFi}$. However, for a proactive $\mathrm{HO}$ roaming and selection decision scheme, whenever WiFi access network is available nearby, the operator should regulate every UE to offload some or all of its traffic through WiFi AP depending on the network condition. In order to effectively offload mobile traffic from macrocell to WiFi APs in heterogeneous network scenario in Figure 1, we need an entity from network side to regulate the cellular UEs when and where WiFi roaming is possible. In addition, it should also provide rightful selection rules for UE. For instance, only authorized APs (AP belonged to operator or authorized provider who has roaming agreement) can be selected. This selectively offloading traffic to legitimated WiFi networks gives mobile operators an opportunity to increase their total network capacity to meet rising traffic demands and a way to extend network coverage and capacity to $\mathrm{WiFi}$ networks. For such scenario, the ANDSF [9] standard framework is a well suitable candidate since it is a $3 \mathrm{GPP}$ approach for controlling handover operation between 3GPP and non3GPP access networks. Since release 8 of 3GPP, 3GPP has specified the ANDSF framework through which the network operator can provide a list of preferred access networks with intersystem mobility policies. However, the drawback of ANDSF's policies is its static characteristic since there is no mechanism to frequently update the policies. As described in [9], for prioritizing AP in selection, the operator assigns AP with an integer number from 1 to 255 (one is the highest priority) and the one with higher priority should be selected by the UE [9]. However, since the condition of the network may vary dynamically from time to time, if the UE only relies on the ANDSF selection rule for selecting new point of service, it can degrade the end-user experience in some cases (e.g., selected AP is too far away or congested one). Therefore, it is also necessary to take into account the real-time network condition at the UE side before making handoff decision. Among various network KPIs for network selection [14, 15] (such as bandwidth, delay, jitter, and latency), we consider the realtime load of WiFi AP is very important to deliver good user experience. Whether this metric is high or low could greatly affect user's experience because the channel load of AP correlates to the available bandwidth as well as delay or latency of the connection that the AP can offer. As far as we are concerned, the channel load information is first defined in IEEE 802.11k, e [25] and later included in the IEEE 802.11u or HS2.0 [11] specification. The UE can obtain this information piece via the beacon message or probe response from supported AP candidates. Since the HS2.0 is rolling out in near future, obtaining the real-time channel load of AP becomes feasible.

For these reasons, our proposed scheme for dynamic $\mathrm{WiFi}$ offloading is designed by following main points:

(i) Taking advantage of ANDSF's selection policies to regulate admission to access points, this requirement to prevent selecting unauthorized AP.

(ii) Using thresholds to control and select preferable AP candidate. Using thresholds to control roaming decision and select the preferable AP. The thresholds are managed by network operator and distributed to UEs via ANDSF MO. We use a defined object in ANDSF MO [9] so-called MaximumBSSLoadValue for traffic load threshold and we also propose an additional threshold for signal quality, MinimumBSSRSSValue.

(iii) Taking into account the load condition of AP obtained from AP candidates (we assume that the WiFi APs are compatible with either HS2.0 or IEEE 802.11k, $\mathrm{u}$ ) to decide whether $\mathrm{WiFi}$ offloading is relevant and which AP is preferable.

3.2. Proposal for Enhancing ANDSF WLANSP Management Object. The ANDSF standard defined various policies such as intersystem mobility, Access Network Discovery Information through the Management Objects (MO), which is a tree based structure. In the latest release 12 [9], a new leaf of ANDSF MO 


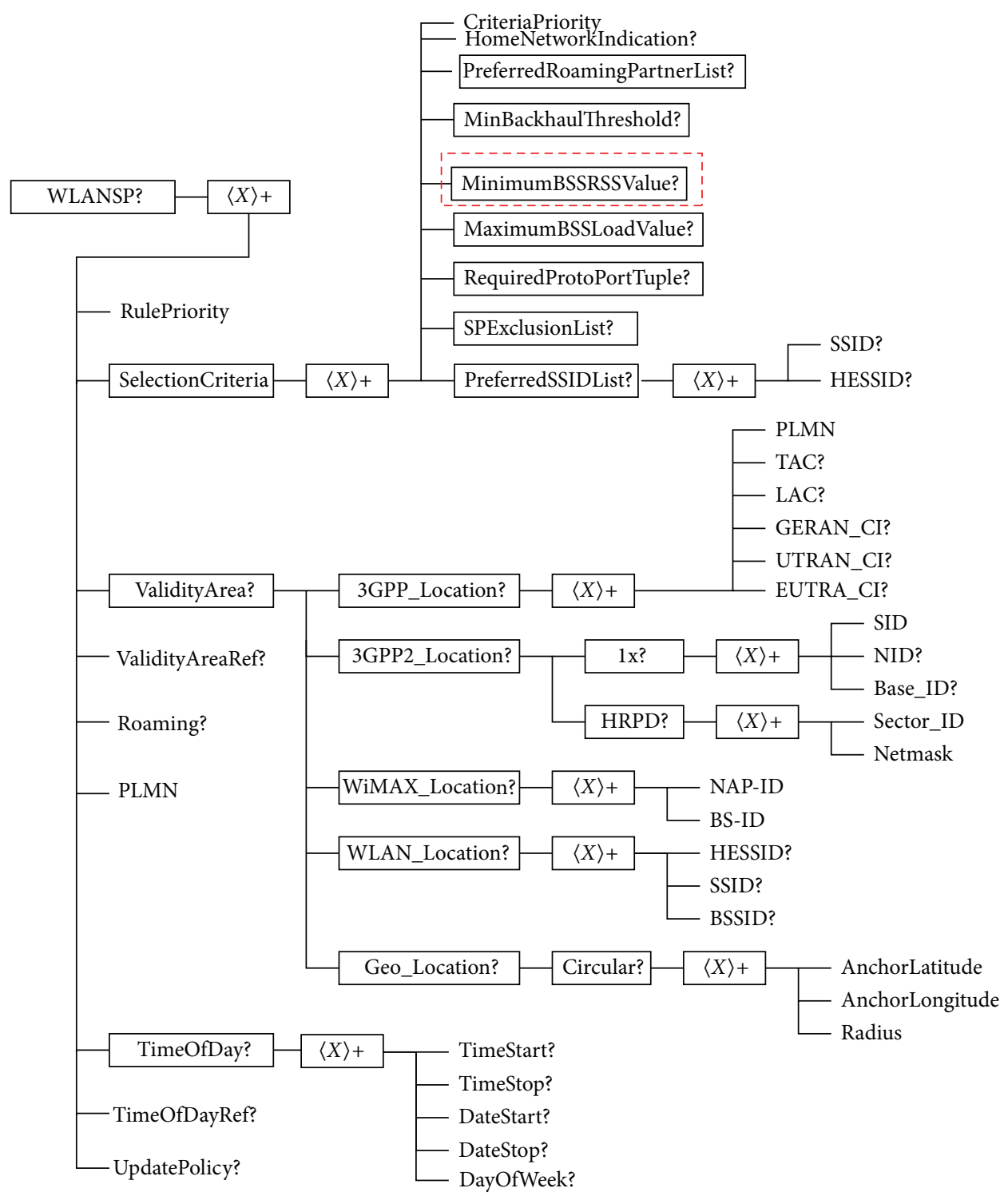

FIgURE 2: Example of enhanced ANDSF OM for WLANSP.

is added for WLAN selection so-called WLANSP (WLAN Selection Policy) (Figure 2). It is a set of operator-defined rules that determine how the UE selects a WLAN access network. The UE is provisioned with WLANSP rules from cellular operator.

Each WLANSP rule includes the following information:

(i) Validity conditions, that is, conditions indicating when the provided rule is valid (the validity conditions can include the time of day, geolocation, and network location (e.g., PLMN, Location Area).

(ii) One or more groups of WLAN selection criteria in priority order (each group contains one or more criteria that should be fulfilled by a WLAN access network in order to be eligible for selection).

The WLANSP MO can contain a set of one or many WLANSP rules. Each rule can have one or more WLAN selection criteria defined in ANDSF/WLANSP $/\langle X\rangle /$ SelectionCriteria node including following attributes:

(i) CriteriaPriority.

(ii) HomeNetworkIndication.

(iii) PreferredRoamingPartnerList.

(iv) MinBackhaulThreshold.

(v) MaximumBSSLoadValue.

(vi) RequiredProtoPortTuple.

(vii) PreferredSSIDList.

(viii) SPExclusionList.

The UE can obtain these selection rules and use them for WiFi selection procedure. However, in our proposed scheme, for the sake of simplicity, we only consider the MaximumBSSLoadValue criterion for WiFi AP selection because it can 
serve as a load threshold in our selection scheme. If the load condition of AP candidate does not satisfy this threshold, it will be eliminated from the selection process. Therefore, this criterion is introduced in our scheme to preeliminated high load AP that cannot potentially provide desirable QoS. In addition, the threshold can also be used to control the number of UEs performing WiFi offloading. For example, if this load threshold value is properly set, only certain number of UEs can roam to WiFi so that the load threshold condition can be satisfied. The rest of cellular UEs is not allowed to offload because of the load threshold. In contrast, if the maximum load threshold is higher, more UEs can roam to WiFi. Therefore, network operator adjusts this threshold to control the percentage of cellular user roaming to $\mathrm{WiFi}$ for offloading.

Apart from the load metric, we also consider the receiving signal strength (RSS) as another important metric that can affect the UE's experienced QoS. If the AP is far away from the UE, it cannot sustain good QoS for the UE in spite of acceptable load condition. Therefore, in our proposed scheme, we add a RSS threshold to eliminate irrelevant AP candidate. Like the max load threshold, the operator utilizes this threshold to control the number of WiFi roaming UEs. For example, if the RSS threshold is high, only UEs that are near to the AP can roam to $\mathrm{WiFi}$.

However, currently, such RSS threshold is not available in the ANDSF WLANSP MO. Therefore, we propose to append this selection policy node to the same OM leaf of MaximumBSSLoadValue in ANDSF WLANSP MO, $\langle X\rangle /$ WLANSP/ $\langle X\rangle /$ SelectionCriteria/ $\langle X\rangle /$ MinimumBSSRSSValue so that this policy can be distributed to UEs in the same manner. Figure 2 shows the tree based structure of ANDSF WLANSP leaf and an example of how the additional selection node is added to existing ANDSF MO standard. If MinimumBSSRSSValue node is not present or the node is present and empty, the UE will not evaluate the node. If it is available, the UE can use this attribute in our scheme for AP selection.

3.3. Real-Time Channel Load for WiFi Access Point. According to Hotspot 2.0 or IEEE 802.11u specification [11], the channel load metric of WiFi AP is defined as one of the new QoS network metrics appended to beacon message of WiFi AP. This channel load metric is inherited from legacy standard IEEE 802.11k [25] ratified in 2008.

The channel load indicates the channel occupancy status of AP. If the load is too high, it can significantly degrade the end-user experience since it increases the contention probability among cochannel UEs. According to [25], the channel utilization or channel load $U_{c}$ is defined as the percentage of time, linearly scaled with 255 representing $100 \%$, that the AP sensed the medium was busy, as indicated by either physical or virtual carrier sense (CS) mechanism. By this definition, this metric indicates the real-time load status of radio channel

$$
\begin{aligned}
U_{c} & =\text { Integer }\left(\frac{t_{\text {busy }}}{T_{M}}\right) \times 255, \\
T_{M} & =t_{c} \cdot t_{b} \cdot 1024
\end{aligned}
$$

The channel load is calculated by (1) and the Channel_Busy Fraction $t_{\text {busy }}$ is defined as the number of microseconds while the physical or virtual carrier sense mechanism in MAC layer keeps indicating busy channel status. The Measurement_Duration $T_{M}$ represents the number of consecutive beacon intervals while the channel busy time is measured. $T_{M}$ is calculated by (2) and $t_{c}$ is the channel utilization measured interval representing the number of consecutive beacon intervals during which the channel busy time is measured. $t_{b}$ is the beacon period value in IEEE802.11-time unit (TU). One TU is 1024 microseconds. The AP regularly calculates its channel load metric every $T_{M}(\mu \mathrm{s})$ and the UEs obtain this channel load metric via either beacon message or probe response message.

3.4. Access Point QoS Indicator. In our proposed scheme, we consider both the channel load and the RSS measured from the UE as the most significant metrics for selecting WiFi AP.

The Receiving Signal Strength (RSS) or SINR is the most widely used metric for conventional handover $(\mathrm{HO})$ decision since it is easy to measure and directly relate to the radio channel quality. With strong RSS, the AP can use high channel modulation and coding scheme and the bit rate becomes higher accordingly. Therefore, it is evident that strong RSS is preferable.

The Channel Load. As aforementioned, this metric indicates the real-time load condition of the AP operating channel. In a wireless network, if the load is too high, it will result in poor quality of service (low throughput, high packet loss, or delay). This metric is even more important for WiFi since WiFi uses CSMA/CA for multiple access. The high channel load can result in high contention rate, which dramatically degrades active UE's throughput in the same channel. Therefore, lower load AP is preferable for good QoE

$$
\begin{aligned}
& \overline{R S S}_{i}=\alpha \cdot \operatorname{RSS}_{i}+(1-\alpha) \cdot \overline{R S S}_{i-1}, \\
& A P Q I_{i}=w_{r} \log _{2}\left(\frac{\overline{R S S}_{i}}{R S S_{-} M I N_{i}}\right) \\
& +w_{l} \log _{2}\left(\frac{1 / \text { Channel_Load }}{i}\right)
\end{aligned}
$$

with $w_{r}+w_{l}=1$.

Because the RSS and channel load have different unit and characteristic, we define a normalized metric known as APQI (Access Point Quality Indicator) in order to make it easier to evaluate and compare the metrics between AP candidates.

Firstly, since each of the above metrics has different unit and characteristics, we have to normalize them. In (4), $\overline{R S S}_{i}$ is the mean value of RSS, which is calculated as (3) while the RSS_MIN is minimum received signal strength of WiFi receiver specified by the Network Interface Controller (NIC) vendor. Each vendor of WiFi NIC has its own sensitive RSS value for the receiver. If the RSS is lower than this value, the data packet will be dropped. Therefore, the normalized term $\overline{R S S}_{i} / R S S \_M I N$ indicates how strong the received signal strength is compared to its minimum value. We use 
binary logarithmic function $\log _{2}\left(\overline{R S S}_{i} / R S S \_M I N_{i}\right)$ to reduce the impact of fluctuation of RSS input. By this definition, this value is independent of the vendor specified hardware specification and comparable among different vendor APs.

The channel utilization metric in (3) is defined as the percentage of time when the AP sensed that the radio channel kept indicating busy by either the physical or virtual carrier sense mechanism [25]. This percentage is linearly scaled of integer range [1-255] that corresponded to $0-100 \%$ channel load. By this definition, the term Channel_Load ${ }_{i} / 255$ is the normalized value of the channel load (the Channel_Load is an integer number [1-255]). Here, we apply logarithmic functions, $\log _{2}\left(1 /\left(\right.\right.$ Channel_Load $\left.\left._{i} / 255\right)\right)$ to reduce the rapid fluctuation of Channel_Load ${ }_{i}$ input and make it comparable with normalized RSS. The APQI is the APQI of ith AP defined as in (4). The first term represents the benefit of signal quality of the radio link between the UE and the AP while the second term represents the benefit of real-time channel load condition of AP. In (4), the APQI is inversely proportional to the channel load metric. It shows a trade-off between the signal quality and the traffic load metrics of AP candidate. The significance of each term depended on the weighted values $w_{r} w_{l}$, for link quality and the channel load or channel utilization, respectively.

3.5. Proposed Roaming Decision and Selection Scheme for Mobile Data Offloading. Our roaming decision and selection scheme is a network assisted and UE driven scheme. The UE has a unique position, which allows it to receive both ANDSF's policies and the real-time network condition from AP candidates. Therefore, it is preferable to let the UE make the decision instead of any other entities in the network. The UE decides timing to make roaming decision and selects preferable point of service based on ANDSF's policies, UE's measured information, and channel load information from AP candidates. The proposed scheme is a decision-making procedure carried out by the connection manager (CM) of end-user's device. In this paper, the CM is a generic term referred to as a functional component that takes input from user preference, input from ANDSF, and APs, and performs connectivity management and the traffic steering, that is, binding user's application/flow to a radio. The CM decides whether cellular-WiFi roaming is relevant or not and which AP candidate is preferable for better QoS. In the proposed scheme, it is worth noting that the channel load metric of $\mathrm{WiFi} \mathrm{AP}$ is required to decide the preferable candidate. However, it should not interfere with conventional homogenous cellular or WiFi handoff procedure.

Figure 3 illustrates the flowchart of proposed scheme.

Step 1. The CM monitors the QoS of current connection. If the QoS is degrading (the data rate drops to certain threshold), running application or user's preference requests for better QoS, it will trigger the process to find better point of service.

Step 2. The CM contacts the ANDSF server at the core network to fetch the list of legitimate WiFi APs and WLANSP selection rule corresponding to UE's location (cell ID of macrocell or SSID or GPS location if applicable). The ANDSF server returns a list of AP candidates that can be accessible from the UE's location. In addition, MaximumBSSLoadValue and MinimumBSSRSSValue thresholds are also obtained from ANDSF MO WLANSP.

Step 3. By using AP discovery information from ANDSF, the UE only scans the surrounding area to check whether the candidate APs are available because provisioned ANDSF's information may be out of date or unreachable from UE's location. By doing this step, UE eliminates unreachable AP and obtains the RSS and the channel load of each candidate $A P$. We assume that the candidate APs support either HS 2.0 or IEEE $802.11 \mathrm{k}$, e. so that the real-time load and the RSS of each AP can be obtained from the beacon message or via ANQP protocol in case of HS.2.0.

Step 4. The CM obtains the list of APs that fulfil the load and RSS thresholds. The thresholds are specified by network carrier via ANDSF's WiFi selection policies. As aforementioned, the MaximumBSSLoadValue is used to select WiFi AP; if the load of AP is higher than this value, it will be eliminated. We introduce the load threshold in order to preeliminate unsuitable AP, which cannot guarantee a good QoS. The MinimumBSSRSSValue can serve as the RSS threshold to eliminate irrelevant AP candidates.

Step 5. If there is no qualified AP after Step 4, the UE will stay at the current network. The UE conducts the conventional homogenous handoff procedure if it is favourable. If these are qualified APs available, the CM calculates the APQI metric (4) for each AP candidate. If the UE is using $\mathrm{WiFi}$, it will calculate the APQI of the associating AP. The AP with highest $A P Q I$ is selected as the candidate for the next point of service. After deciding the preferable AP, the CM starts the handoff procedure to selected AP.

Step 6. If there is no better point of service, the UE will stay at the current network. The CM returns to Step 1.

The pseudocode of our proposed scheme is shown in Algorithm 1.

\section{Simulation and Results}

4.1. Simulation Model Preparation. Although there are several adoptions of ANDSF [9] standard for commercial solutions for network selection in heterogeneous network [26], as far as we are concerned, there is no simulation of ANDSF standard for academic studies. Therefore, in order to evaluate our proposed scheme, we developed a system model of HetNet with vertical handoff (VHO), ANDSF server/client, and connection manager running on each mobile node. The whole system model is developed by NCTUns 6.0, which is a powerful open source network simulator developed by National Chiao Tung University [27-30]. It can simulate varieties of popular wired and wireless network entities and protocols such as IEEE WiFi $802.11 \mathrm{a} / \mathrm{b} / \mathrm{g}$, ad hoc networks, WiMAX IEEE $802.16 \mathrm{e} / \mathrm{d} / \mathrm{p}$, multiple-interface UE, GPRS, 


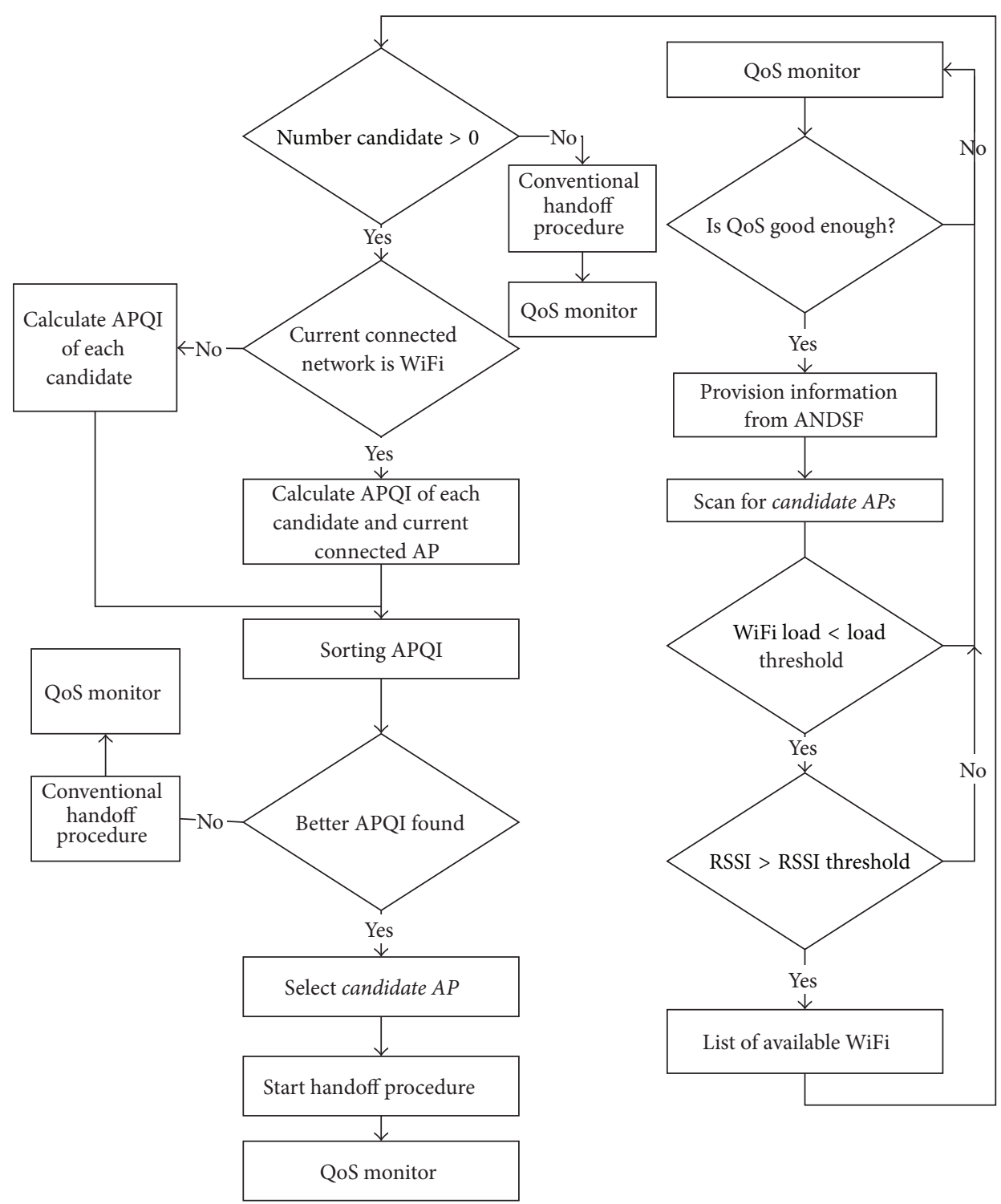

FIgURE 3: Proposed roaming decision and point of service selection scheme.

and satellite and vehicular networks. However, one of the key features of this simulator software is that it allows running user-defined $\mathrm{C} / \mathrm{C}++$ application on the simulated node. In our simulation model, we take advantage of this feature to simulate the ANDSF server and the connection manager (CM) for each UE node.

ANDSF server is a $C$ program, which provisions information of available APs, load threshold, and RSS threshold. The CM is also C program running on each mobile node that executes our proposed scheme. It can obtain the RSS and the real-time channel load of AP candidate through MAC layer. The CM contacts the ANDSF host to fetch policies and thresholds via UDP socket. In addition, the CM can also obtain the RSS and the channel load of AP candidate from the MAC layer. As the simulation tool does not support IEEE 802.11k, e or HS 2.0, we modify the MAC layer of the WiFi AP node to append the real-time channel load information into the beacon message. In (1), the channel busy fraction is defined as the number of microseconds when the physical or virtual carrier sense indicates the channel is busy. In our simulation, we use the virtual carrier sense, Request to Send/Clear to Send (RTS/CTS) mechanism for detecting busy channel [25]. In [25] the default value of "channelUtilizationBeaconIntervals" in (1) is set to 50. However, this parameter is set to 10 in our simulation and the beacon broadcast interval is $100 \mathrm{~ms}$.

The coefficients $w_{r}$ and $w_{l}$ are the weight values of RSS and channel load metric, respectively, used to calculate the APQI. In our simulation, we notice that if $w_{r} \gg w_{l}$, the system performs much like conventional WiFi selection based on RSS. Therefore, for the scope of this simulation, we choose $w_{r}=0.4$ and $w_{l}=0.6$.

Although the simulator software supports multiple-interface mobile UE and horizontal handover within WiMAX 
Input: Set the parameters: RSS, RSS_Min, Channel_load, MaximumBSSLoadValue, MinimumBSSRSSValue, $w_{r}, w_{l}$ Output: AP candidate.

(1) While (1) do \{

(2) if QoS degrading trigger then

(3) Obtain data from ANDSF server

(a) Obtain AP candidates Information (SSID, operating channel)

(b) Obtain Load and RSS thresholds MaximumBSSLoadValue, MinimumBSSRSSValue

(4) qualified_AP_List $=$ nil;

for each $A P_{i}$ in $A P$ candidate List \{

(a) Obtain information of available $A P_{i}$ information from physical Layer,

(b) Obtain $R S S_{i}$, calculate $\overline{R S S}_{i}$ (3).

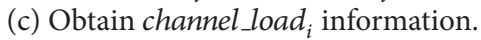

(d) if $\overline{R S S}_{i}>$ MinimumBSSRSSValue \&\& channel load ${ }_{i}<$ MaximumBSSLoadValue then qualified_AP_List add AP \}

If qualified_AP_List is empty then return;

(5) Candidate_AP $\rightarrow$ APQI = calculate $A P Q I_{1}$ using (4);

for each $A P_{i}$ in $A P$ candidate qualified_AP_List \{

calculate $A P Q I_{i}$ using (4)

if $A P_{i} \rightarrow A P Q I>$ Candidate $\_P P \rightarrow A P Q I$ then Candidate $A P=A P_{i}$;

\}

(6) if Candidate $A P !=$ current AP then

return Candidate_AP;

\}

Algorithm 1: Roaming decision and preferable AP selection.

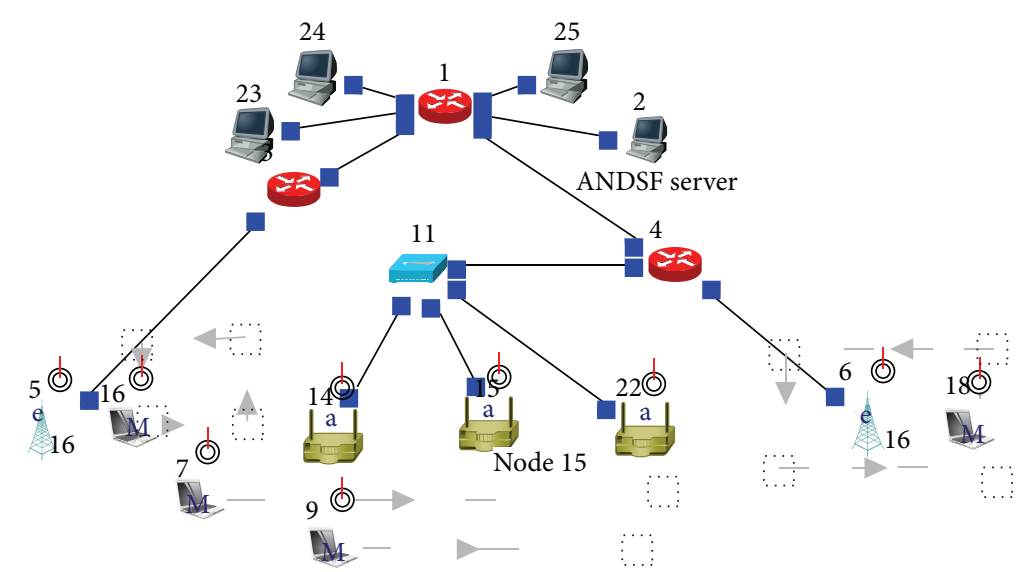

FIGURE 4: Simulation scenario setup for HetNet with interworking WiMAX BSs, WiFi APs, and Multiple Interfaces UEs.

or WiFi, it does not support VHO between WiMAX BS and WiFi AP. Therefore, we modify the source code of the simulation software to simulate the vertical handover between macrocell (WiMAX IEEE 802.16e) and WiFi IEEE 802.11a. It is worth noting that our proposed scheme is proposed for 3GPP cellular (LTE or UMTS) and WiFi. However, the simulation software NCTUns version 6.0 [27] does not support LTE. Therefore, we have to use WiMAX instead of LTE for our HetNet model. Since our proposed scheme does not consider any metric from the macrocell, using WiMAX instead of LTE for access network does not cause any difference in the simulation result. In addition, due to a problem related to mobile IP protocol of the simulator, the simulation of VHO is not a seamless handover. Therefore, the UE's connections are interrupted when performing the VHO.
4.2. Simulation Model Description. Figure 4 shows the screenshot of our simulation setup in the simulator and the simulation parameters are listed in Table 1 . This setup is a typical WiFi deployment scenario to offload traffic from cellular network as we described in Figure 1. We arrange a typical WiMAX-WiFi interworking scenario with two IEEE 802.16e BS1, 2 (Nodes 5, 6), three 802.11a WiFi APs 1, 2, and 3 (Nodes 14, 15, and 22) and four multiple wireless interface UEs 1, 2, 3, and 4 (Nodes 7, 9, 16, and 18 consecutively) equipped with both WiMAX and WiFi 802.11a interfaces. All of WiMAX BSs and WiFi APs are interconnected via routers and switches, which is a simplified core network in Figure 1. We simulate the typical movement pattern of UEs when they move from the coverage of WiMAX to WiFi. During the simulation, UEs 1, 2, and 3 move from the 
TABLE 1: Simulation parameter.

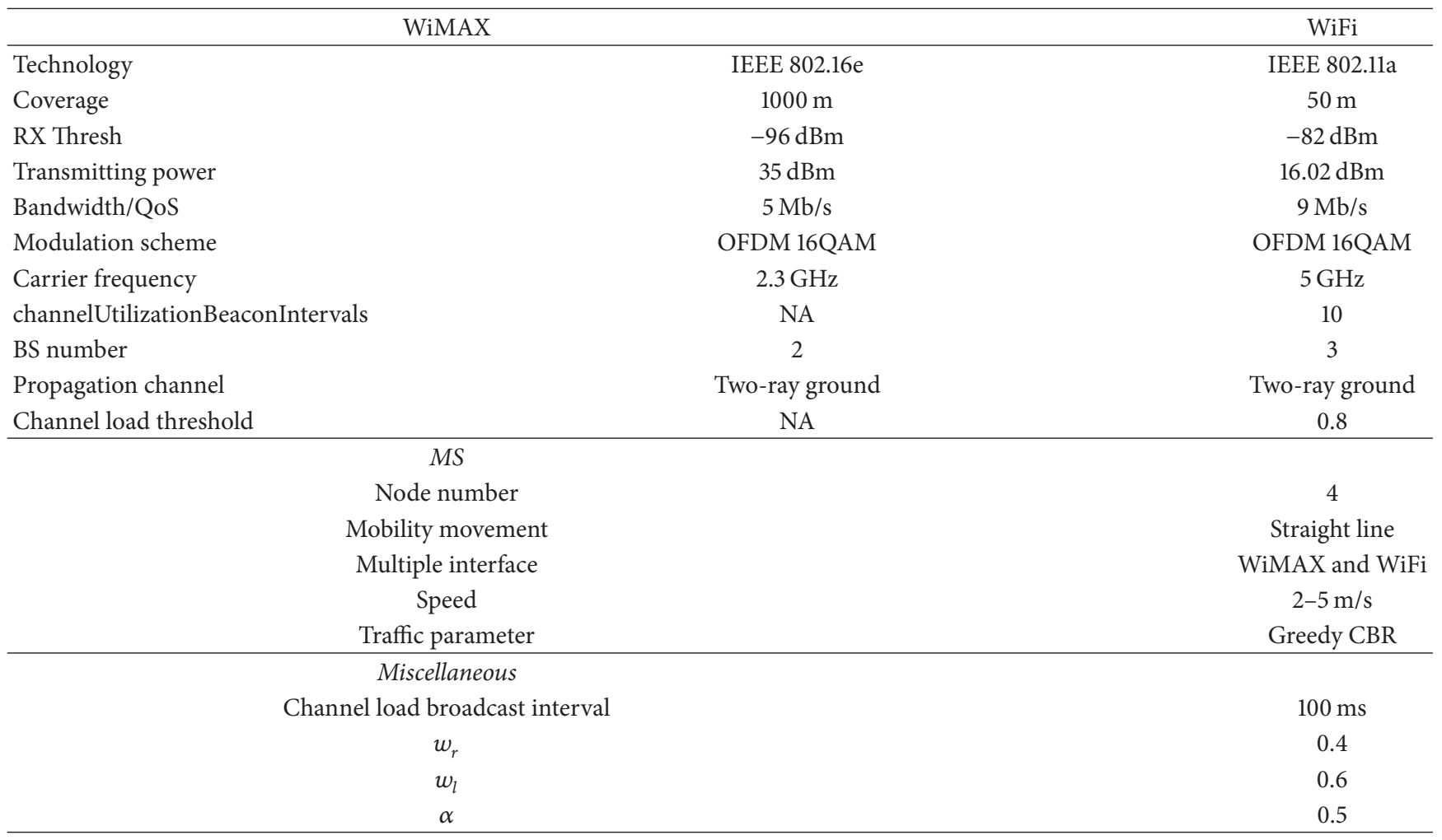

coverage of BS1 toward WiFi coverage area while the UE4 (node 18) moves around BS2. All of the UEs have a CM application embedded with our proposed scheme to monitor and manage their connectivity. The ANDSF host (Node 2) resides at network side and it is reachable by all of UEs. The ANDSF communicates with the UE's CM to provide WiFi AP candidates as well as load and RSS threshold. In order to simulate WiFi roaming trigger event when QoS is not good enough, we randomly disrupt the WiMAX base station connection during the simulation.

At the beginning, the UEs are connected via WiMAX BSs; UEs 1, 2, and 3 (Nodes 7, 9, and 16) are associated with BS1 while UE4 (Node 18) is associated with BS2. At $T=4 \mathrm{~s}$, the UEs start sending greedy CBR (Constant Bit Rate) toward the Correspondent Node (CN) (Nodes 2, 23, 24, and 25 in Figure 4). Figures 5 and 6 show the traffic of BS1, BS2. The throughput of BS1 and BS2 gradually increases and reaches the maximum bit rate at $1975 \mathrm{~KB} / \mathrm{s}$ and $659 \mathrm{~KB} / \mathrm{s}$, respectively.

At $T=10 \mathrm{~s}$, the CM of UE2 fetches information from ANDSF policies, which contains AP candidates 1, 2, and 3 (Nodes 14, 15, and 22). Subsequently, it scans the surrounding area to obtain the channel load and RSS of each AP candidate. The CM calculates the APQI of each AP according to (4). The UE2 picks AP1 because it has the highest APQI. The UE2 starts VHO procedure to AP1 at $T=10 \mathrm{~s}$. As showed in Figures 5 and 7 the traffic of BS1 (red line) drops to $1316 \mathrm{~KB} / \mathrm{s}$ at $T=11 \mathrm{~s}$ while the traffic of AP1 is gradually rising at $T=$ $13 \mathrm{~s}$ when the VHO is completed.

At $T=12 \mathrm{~s}$, the CM of UE1 (Node 9) detects the QoS of WiMAX BS degraded; it sends a request to ANDSF host to

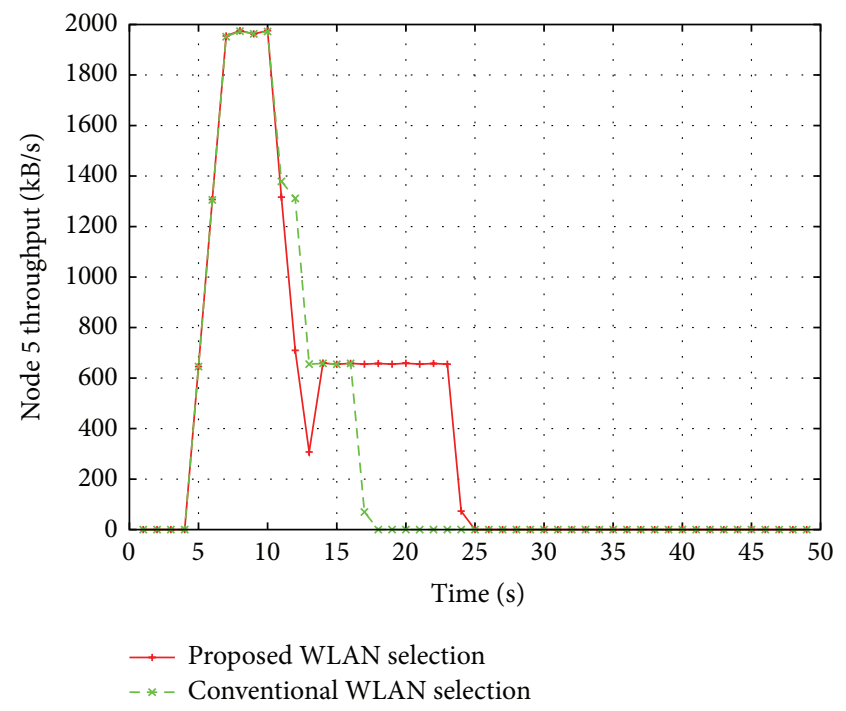

Figure 5: WiMAX BS1 (Node 5) throughput.

obtain the candidate list. The host returns the list of available APs 1, 2, and 3 (Nodes 14, 15, and 22). The UE1 carries out the same procedure as the UE2 to calculate the APQI of each AP candidate. The UE1 selects and starts VHO procedure to AP2 at $T=12 \mathrm{~s}$. Figure 5 shows that the traffic of BS1 continual drops to $659.5 \mathrm{~KB} / \mathrm{s}$ at $T=14 \mathrm{~s}$. From Figure 8 , at $T=16 \mathrm{~s}$, the traffic of AP2 starts rising when the handover procedure of UE1 is completed. 


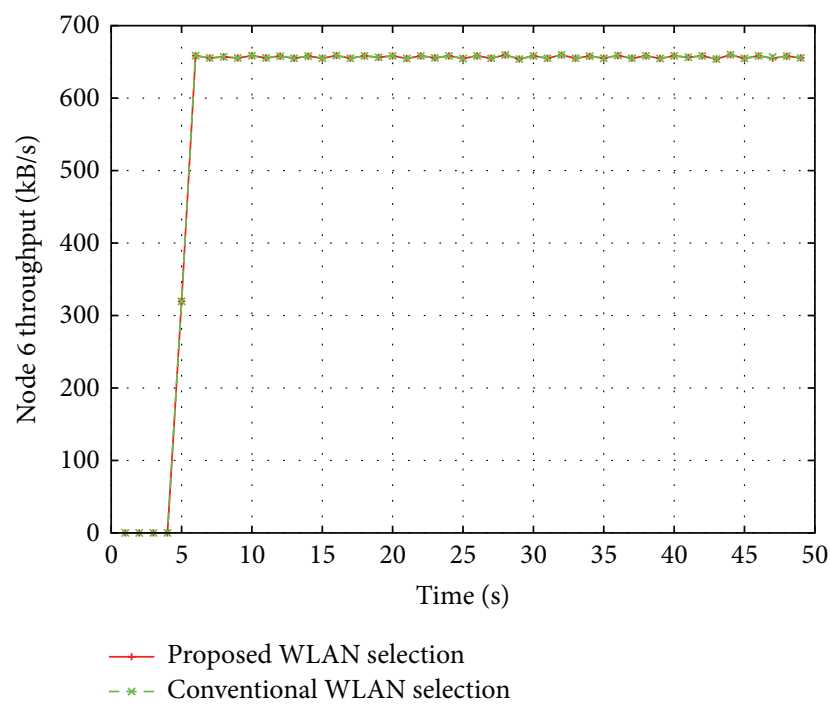

Figure 6: WiMAX BS2 (Node 6) throughput.

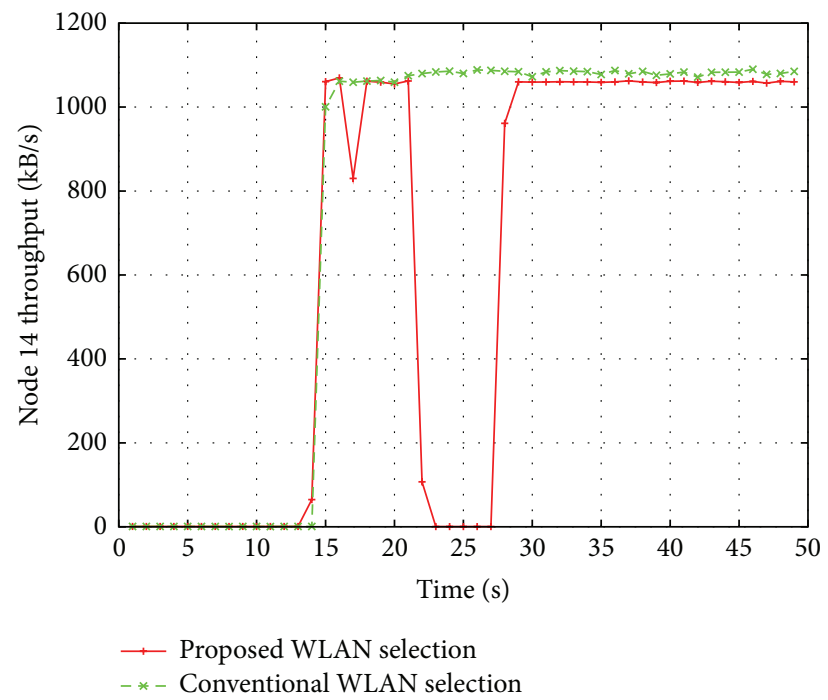

Figure 7: WiFi AP1 (Node 14) throughput.

At $T=15 \mathrm{~s}$, the UE3 (Node 16) carries out the same procedure as UE1 and UE2. However, AP1 and AP2 are serving UE1 and UE2 and the channel load threshold is not satisfied. The UE3's CM decides to keep the connection with WiMAX BS1 since the specified APs are busy. As showed in Figure 4, from $T=14 \mathrm{~s}$ the throughput of $\mathrm{BS} 1$ remains at $650 \mathrm{~KB} / \mathrm{s}$ which is the traffic of UE3.

At $T=20 \mathrm{~s}$, the UE1 moves closer to the AP3. The RSS of AP3 satisfies the RSS threshold. The UEl's CM decides to switch the connection to AP3. In Figure 9, the traffic of AP3 is raising from $T=20 \mathrm{~s}$ while the traffic of AP2 in Figure 8 drops at the same time.

At $T=21 \mathrm{~s}$, the CM of UE2 calculates the APQI of each AP candidate. The AP2 becomes a better candidate for UE2 since it remains idle and closer to UE2. The UE2 decides to select AP2 as the target AP for handoff. In Figure 7, the traffic

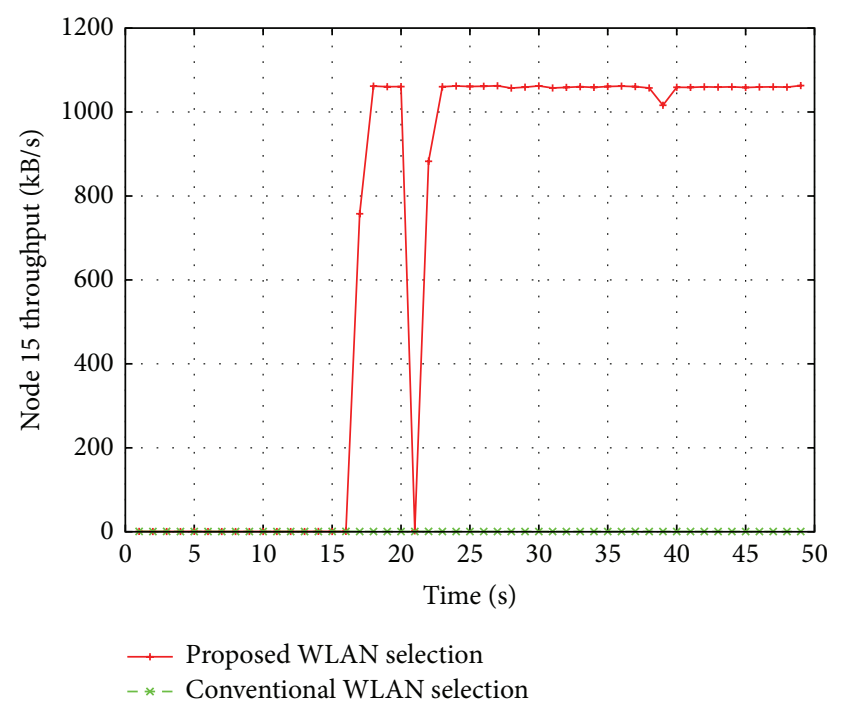

Figure 8: WiFi AP2 (Node 15) throughput.

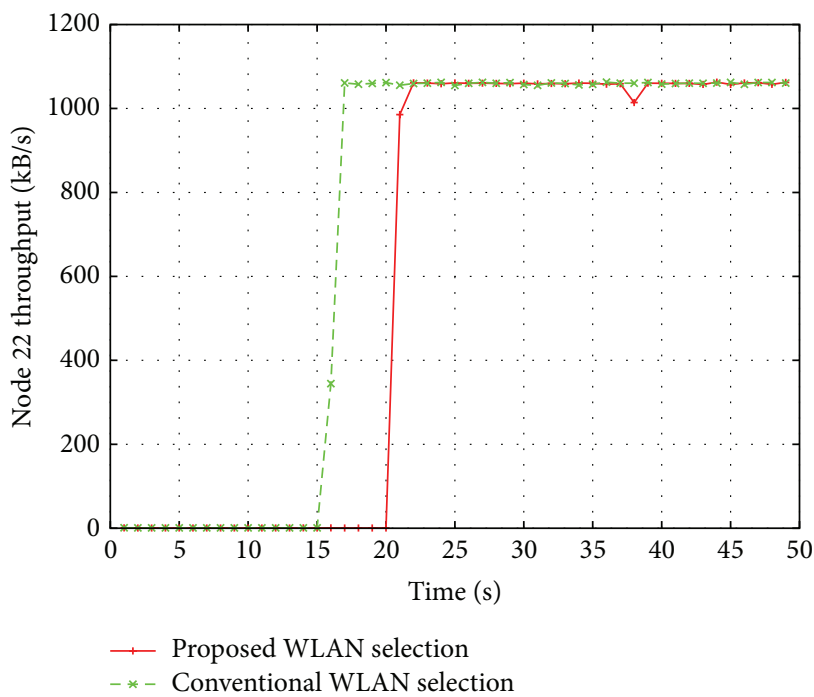

Figure 9: WiFi AP3 (Node 22) throughput.

of AP1 drops and the traffic of AP2 increases at $T=21 \mathrm{~s}$ when the handoff process is going.

At $T=24 \mathrm{~s}$, the UE3 calculates the APQI for each AP candidate. The AP1 becomes the best candidate for UE3 this time because it is idle and satisfies both channel load and RSS threshold. The UE3's CM decides to switch the connection from BS1 to AP1. In Figure 5, the traffic of BS1 drops to $0 \mathrm{~KB} / \mathrm{s}$ at $T=25 \mathrm{~s}$ when all of UEs are transferred to Wi-F APs. In Figure 7, the traffic of AP1 increases from $T=26 \mathrm{~s}$ when handoff process is complete.

The UE4 moves back and forth around the BS2; however there is no available AP candidate at its location. Therefore, the UE4's CM decides to keep the connection with BS2 throughout the simulation.

Figure 10 shows the distribution of UEs at $T=25 \mathrm{~s}$ after transferring from WiMAX BS1; each WiFi AP serves one UE (Figure 11). 


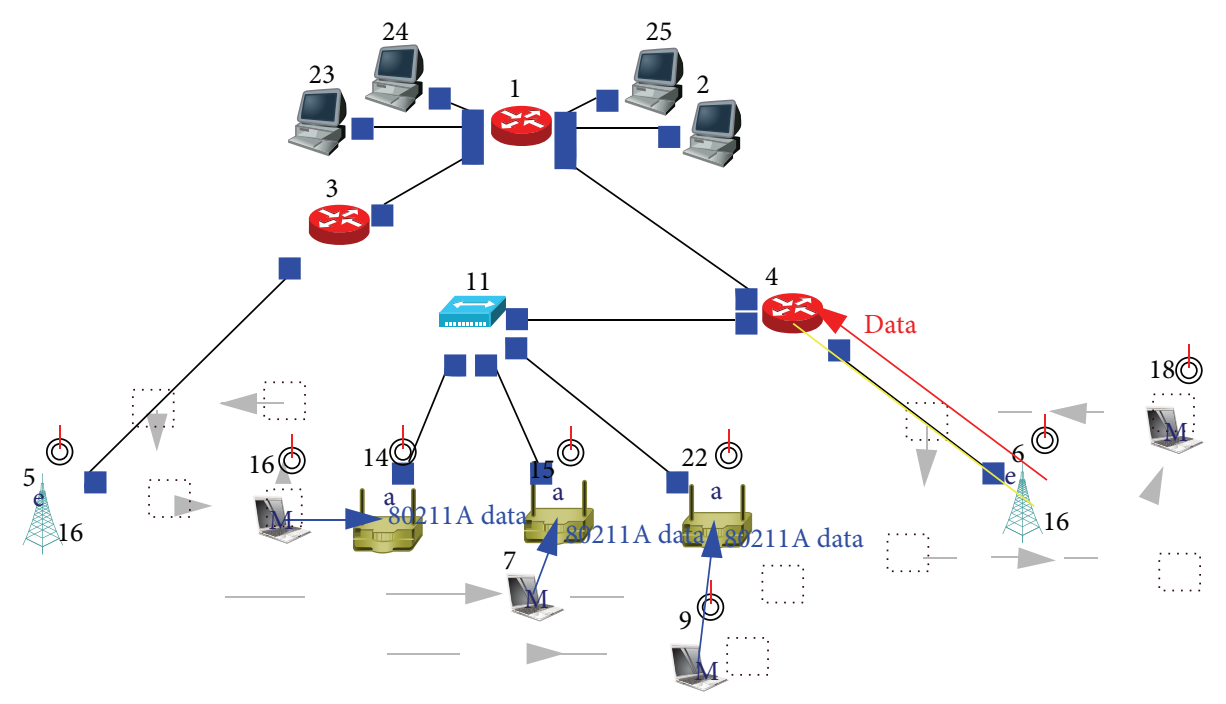

FIGURE 10: Screenshot from simulator for proposed scheme.

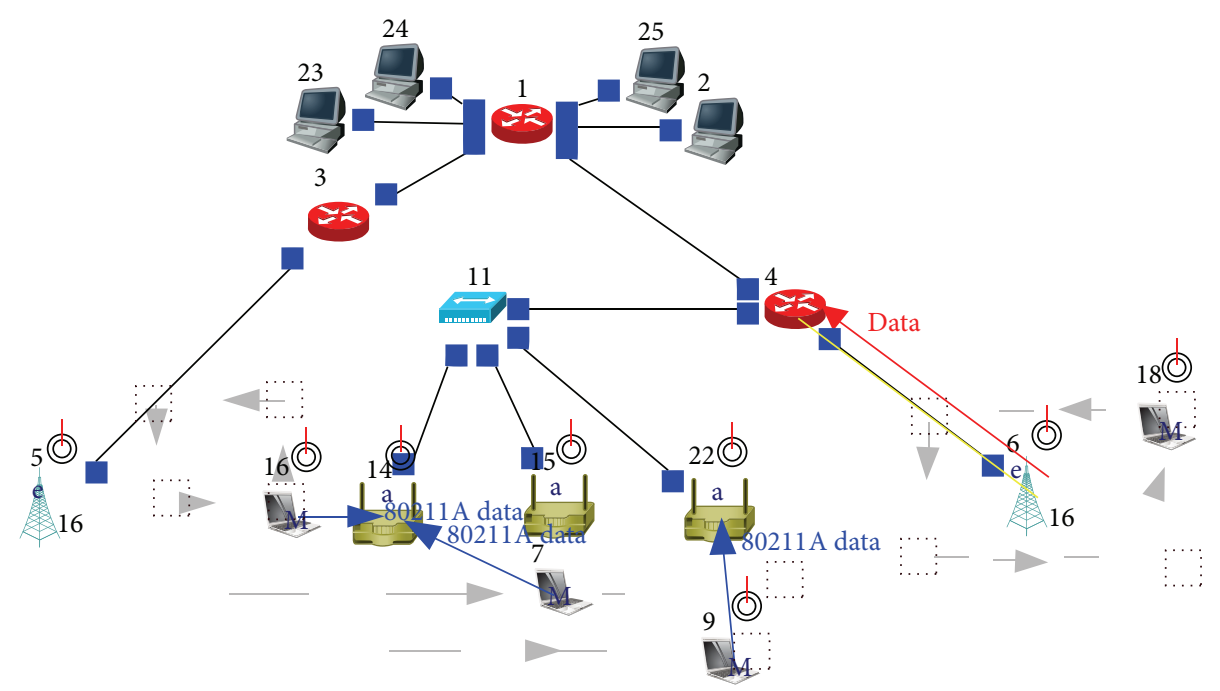

FIGURE 11: Screenshot from simulator with conventional scheme.

4.3. Simulation Result Discussion. In this section, we increased the density of the mobile nodes with random mobility. The simulation model is shown in Figure 12. The density of UEs gradually increases around WiFi APs when UEs move toward them. We compare the performance with that of the conventional WiFi selection scheme. As aforementioned, in the conventional selection based on RSS or SINR, if there are several of available APs, the nearest AP (regardless of AP's load status) will be selected and the connection will remain until the signal strength becomes unacceptable (below RX sensitive threshold).

At the beginning, the UEs are connected via WiMAX BSs. At $T=4 \mathrm{~s}$, the UEs start sending greedy CBR (Constant Bit Rate) toward the Correspondent Node (CN) (Nodes 23, 24, and 25 in Figure 12). Figure 13 shows the traffic of UEs rising at $T=4 \mathrm{~s}$.

At $T=7 \mathrm{~s}$, the CM of UEs fetches discovery and selection policies from ANDSF, which contains AP candidates list, signal strength, and AP's load thresholds corresponding to UEs location. Afterward, the UEs scan the surrounding area to obtain the channel load and RSS of each AP candidate. The AP candidates are evaluated based on their channel load and the received signal strength metrics. Only APs, which satisfy the load threshold and the signal strength threshold, are considered in the next steps. The CM of UEs calculates the APQI metric of each AP. The AP candidates are ranked by the APQI metric and the top AP is selected as AP candidate.

The numerical results show that the proposed scheme outperforms the conventional WiFi selection scheme in terms of overall system throughput or average UE's data throughput. With the knowledge of the network conditions and selection policies, UEs can offload their traffic more efficiently. With our proposed scheme, the UE can proactively decide the right timing for making $\mathrm{WiFi}$ roaming based on the policies from the network and UE's measured information. Furthermore, the UE can also dynamically select preferable 


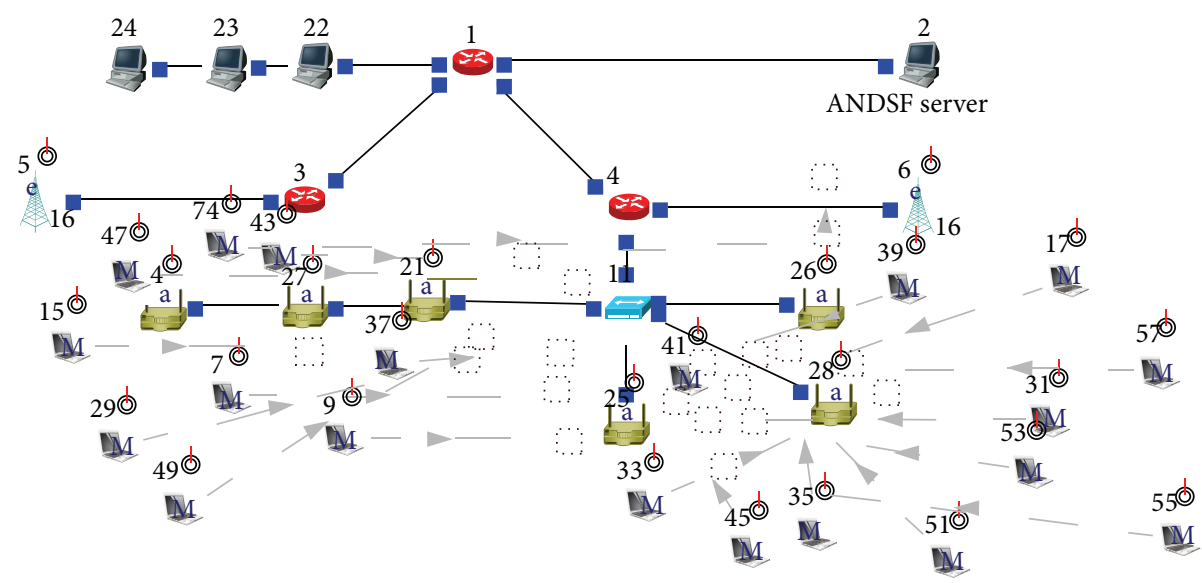

FIgURE 12: Simulation setup with 20 UEs and 6 APs and 2 WiMAX BSs.

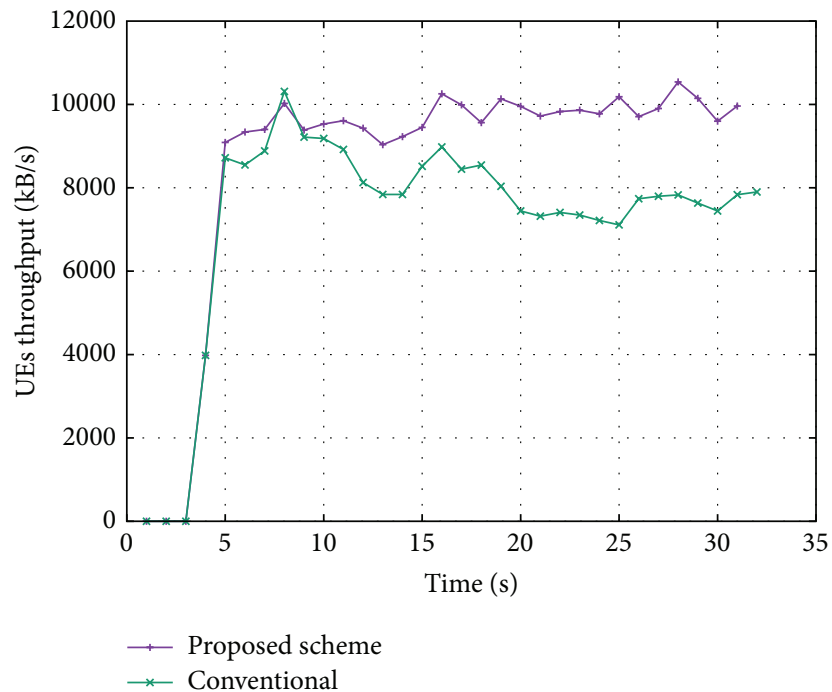

FIGURE 13: Overall system throughput comparison proposed scheme and conventional scheme.

AP (in terms of load condition and radio link condition) when moving. Therefore, the traffic of the macrocells is offloaded and distributed among available APs. It increases the utilization rate of available APs since all of APs are utilized while one AP is left unused in conventional case. In addition, we are also aware that proposed scheme has a drawback since it increases the number of handoffs. This drawback results from the fact that UEs are dynamically switched to preferable AP to optimize their throughput. Therefore, we have a trade-off between the number of handoffs and experienced throughput of mobile node. This problem will be considered in our future work.

\section{Conclusion and Future Work}

Our main contribution in this paper is that we proposed a novel WiFi roaming selection scheme for cellular data offloading. In our proposed scheme, we utilized the channel load metric first defined in IEEE802.11k as well as IEEE 802.11u for the proposed scheme. As far as we are concerned, this channel load has not been considered in any related work. We defined a new metric, APQI, which was used to rank AP candidates based on their channel load status and signal strength. In addition, we utilized a series of signal level and AP load thresholds to eliminate unqualified candidates, which reduce the processing time and reduce unnecessary delay. We proposed to append signal strength threshold into the ANDSF OM WLANSP leaf so that UEs can easily fetch this information piece from ANDSF server for network selection. In addition, we also considered the practical implementation of our proposed scheme. Therefore, we reviewed the state of the art in 3GPP and IEEE and developed our scheme based on the ratified IEEE 802.11 $\mathrm{k}, \mathrm{u}[11,25]$ and 3GPP TS 23.312 [9] standard so that it can be applicable in future wireless network. In this paper, we also introduced our simulation model of ANDSF and vertical handover. Unlike previous works, our simulation model is an end-to-end model from application to physical layer. We took into account user's mobility and realistic traffic model. During the simulation process, the ANDSF server and the UE's connection manager entities behave and interact corresponding to real-time network events. By using this simulation model, we evaluated the proposed scheme in a typical heterogeneous network scenario with interworking macrocell (WiMAX) and WiFi.

We demonstrated how the proposed scheme performs in a typical HetNet scenario with WiFi APs and macrocells. The result showed that our proposed scheme dynamically steered the UE's traffic from macrocell and is distributed to available WiFi APs. As a result, both the overall system throughput and the utilization of available WiFi APs were improved. In addition, we observed better user's experienced throughput compared to conventional scheme. In our future work, we will further enhance the proposed scheme and evaluate the proposed scheme in more complex network scenario. In this paper, the network is assumed to support only data-like services. Other types of services such as voice or a combination of different services could also be studied. 
A more realistic user behaviour model could be employed. The robustness of the results can be analysed by varying some system parameters such as hotspot radius and hotspot position. In addition, we will also consider the UE's context with different QoS requirements.

\section{Conflict of Interests}

The authors declare that there is no conflict of interests regarding the publication of this paper.

\section{References}

[1] W. Doug, "Announcing the Cisco Visual Networking Index Mobile Forecast, 2010-2015," April 2015, http://blogs.cisco.com/ tag/mobile-data-forecast.

[2] D. Cavalcanti, D. P. Agrawal, C. Cordeiro, B. Xie, and A. Kumar, "Issues in integrating cellular networks, WLANs, and MANETs: a futuristic heterogeneous wireless network," IEEE Wireless Communications, vol. 12, no. 3, pp. 30-41, 2005.

[3] R. Chakravorty, P. Vidales, K. Subramanian, I. Pratt, and J. Crowcroft, "Performance issues with vertical handoversexperiences from GPRS cellular and WLAN hot-spots integration," in Proceedings of the 2nd IEEE International Conference on Pervasive Computing and Communications (PerCom '04), pp. 155-164, March 2004.

[4] K. Murray, R. Mathur, and D. Pesch, "Intelligent access and mobility management in heterogeneous wireless networks using policy," in Proceedings of the ACM 1st International Workshop on Information and Communication Technologies, pp. 181$186,2003$.

[5] K.-S. Kong, W. Lee, Y.-H. Han, M.-K. Shin, and H. You, "Mobility management for all-IP mobile networks: mobile IPv6 vs. proxy mobile IPv6," IEEE Wireless Communications, vol. 15, no. 2, pp. 36-45, 2008.

[6] H. Fathi, S. S. Chakraborty, and R. Prasad, "Optimization of mobile IPv6-based handovers to support VoIP services in wireless heterogeneous networks," IEEE Transactions on Vehicular Technology, vol. 56, no. 1, pp. 260-270, 2007.

[7] C. Guo, Z. Guo, Q. Zhang, and W. Zhu, "A seamless and proactive end-to-end mobility solution for roaming across heterogeneous wireless networks," IEEE Journal on Selected Areas in Communications, vol. 22, no. 5, pp. 834-848, 2004.

[8] 3GPP. 3rd Generation Partnership Project, "Architecture enhancements for non-3GPP accesses (release 12)," Technical Specification 3GPP TS 23.402, 2014.

[9] Generation Partnership Project, "Access network discovery and selection function management object (release 12)," Technical Specification 3GPP TS 24.312; V12.4.0, 2014.

[10] IEEE, "IEEE standard for information technology-telecommunications and information exchange between systems-local and metropolitan area networks-specific requirements, part 11: wireless LAN Medium Access Control (MAC) and Physical Layer (PHY) specifications," IEEE Std 802.11, IEEE, 2012.

[11] Wi-Fi Alliance, "Hotspot 2.0 Technical Specification Package (Release 2); v1.0.0”.

[12] 4G Americas, Integration of Cellular and WiFi Networks, 4G Americas, 2013.
[13] M. Zekri, B. Jouaber, and D. Zeghlache, "A review on mobility management and vertical handover solutions over heterogeneous wireless networks," Computer Communications, vol. 35, no. 17, pp. 2055-2068, 2012.

[14] M. Kassar, B. Kervella, and G. Pujolle, "An overview of vertical handover decision strategies in heterogeneous wireless networks," Computer Communications, vol. 31, no. 10, pp. $2607-$ 2620, 2008.

[15] J. Fu, J. Wu, J. Zhang, L. Ping, and Z. Li, "A novel AHP and GRA based handover decision mechanism in heterogeneous wireless networks," in Information Computing and Applications: First International Conference, ICICA 2010, Tangshan, China, October 15-18, 2010. Proceedings, vol. 6377 of Lecture Notes in Computer Science, pp. 213-220, Springer, Berlin, Germany, 2010.

[16] H. Silva, L. Figueiredo, C. Rabadão, and A. Pereira, "Wireless networks interoperability-wifi wimax handover," in Proceedings of the 4th International Conference on Systems and Networks Communications (ICSNC '09), pp. 100-104, IEEE, Porto, Portugal, September 2009.

[17] 3GPP Study on Wireless Local Area Network (WLAN) 3GPP Radio Interworking, "Technical specification group radio access network," 3GPP TR 37.834, 2013, (Release 12).

[18] D. H. Hagos and R. Kapitza, "Study on performance-centric offload strategies for LTE networks," in Proceedings of the 6th Joint IFIP Wireless and Mobile Networking Conference (WMNC '13), pp. 1-10, IEEE, Dubai, United Arab Emirates, April 2013.

[19] B. H. Jung, N.-O. Song, and D. K. Sung, "A network-assisted user-centric WiFi-offloading model for maximizing per-user throughput in a heterogeneous network," IEEE Transactions on Vehicular Technology, vol. 63, no. 4, pp. 1940-1945, 2014.

[20] J. Lee, Y. Yi, S. Chong, and Y. Jin, "Economics of WiFi offloading: trading delay for cellular capacity," IEEE Transactions on Wireless Communications, vol. 13, no. 3, pp. 1540-1554, 2014.

[21] P. S. Bithas, A. S. Lioumpas, G. K. Karagiannidis, and B. S. Sharif, "Interference minimization in hybrid WiFi/cellular networks," in Proceedings of the 19th IEEE International Workshop on Computer Aided Modeling and Design of Communication Links and Networks (CAMAD '14), pp. 198-202, IEEE, Athens, Greece, December 2014.

[22] IEEE, IEEE Standard for Local and Metropolitan Area Networks. Part 21: Media Independent Handover Services, IEEE, New York, NY, USA, 2009.

[23] M. Kassar, B. Kervella, and G. Pujolle, "An overview of vertical handover decision strategies in heterogeneous wireless networks," Computer Communications, vol. 31, no. 10, pp. 26072620, 2008.

[24] O. Khattab and O. Alani, "A survey on media independent handover (MIH) and IP multimedia subsystem (IMS) in heterogeneous wireless networks," International Journal of Wireless Information Networks, vol. 20, no. 3, pp. 215-228, 2013.

[25] IEEE, "IEEE standard for information technology-telecommunications and information exchange between systems-local and metropolitan area networks-specific requirements, part 11: wireless LAN Medium Access Control (MAC) and Physical Layer (PHY) specifications amendment 1: radio resource measurement of wireless LANs," IEEE Std 802.11k-2008, version 1.2.1, 2008.

[26] Green Packet Mobile data offload solution using ANDSF, April 2015, http://www.greenpacket.com/solutions/dynamic-WiFi-offload.html. 
[27] S. Y. Wang, C. L. Chou, and C. C. Lin, "The design and implementation of the NCTUns network simulation engine," Simulation Modelling Practice and Theory, vol. 15, pp. 57-81, 2007.

[28] S.-Y. Wang, P.-F. Wang, Y.-W. Li, and L.-C. Lau, "Design and implementation of a more realistic radio propagation model for wireless vehicular networks over the NCTUns network simulator," in Proceedings of the IEEE Wireless Communications and Networking Conference (WCNC '11), pp. 1937-1942, Cancun, Mexico, March 2011.

[29] S. Y. Wang and P. F. Wang, "NCTUns 6.0: a simulator for advanced wireless vehicular network research," in Proceedings of the IEEE 71st Vehicular Technology Conference (VTC-Spring '10), pp. 1-2, Taipei, Taiwan, May 2010.

[30] S. Y. Wang and R. M. Huang, "NCTUns tool for innovative network emulations," in Computer-Aided Design and Other Computing Research Developments, pp. 155-183, Nova Science, 2009. 

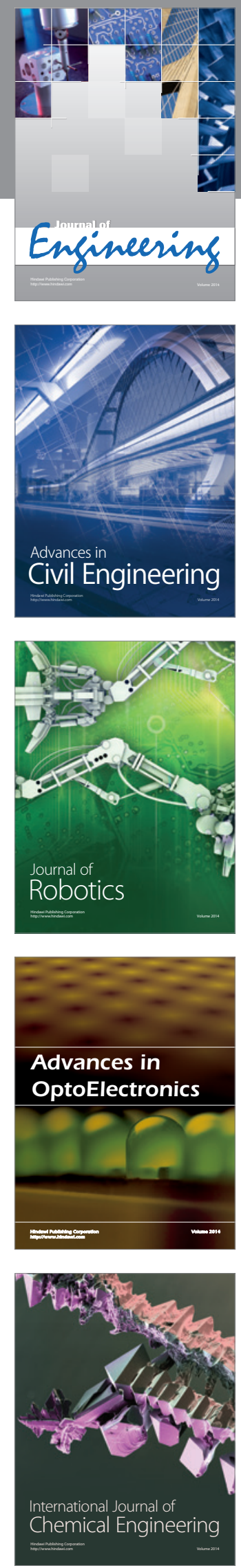

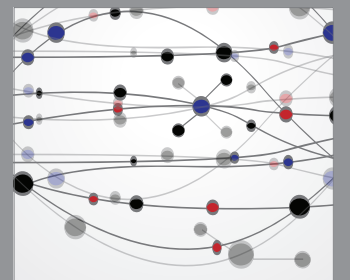

The Scientific World Journal
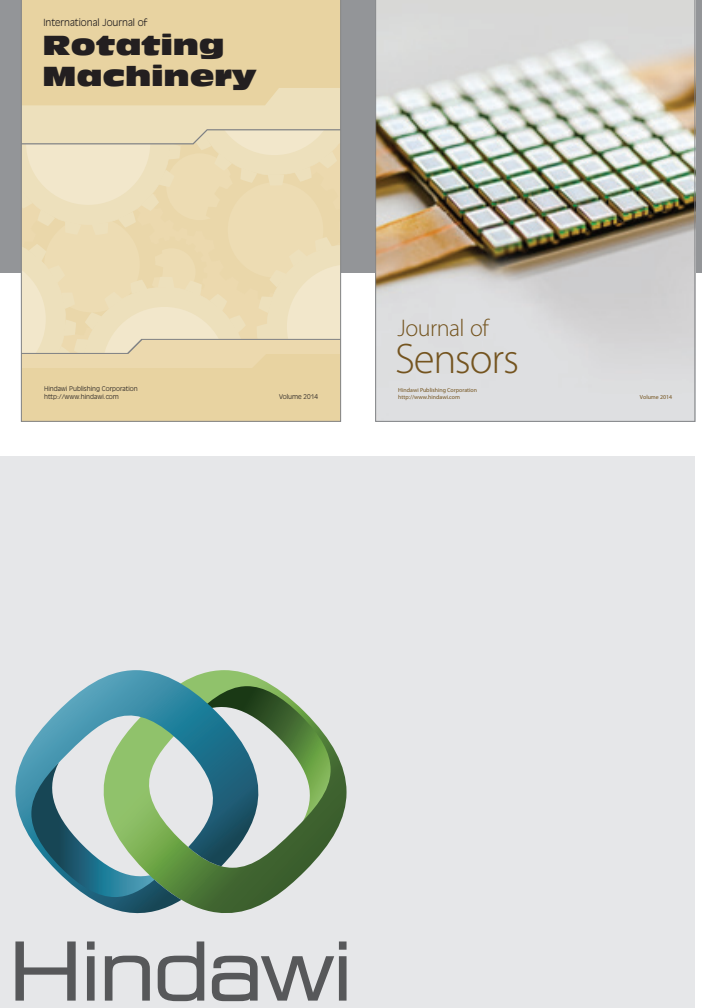

Submit your manuscripts at http://www.hindawi.com
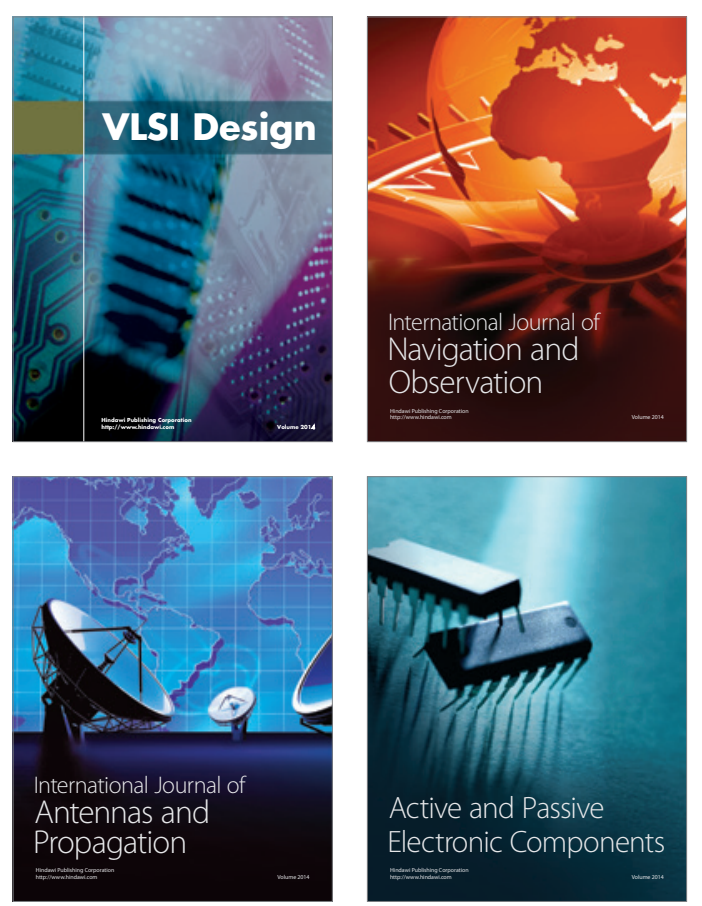
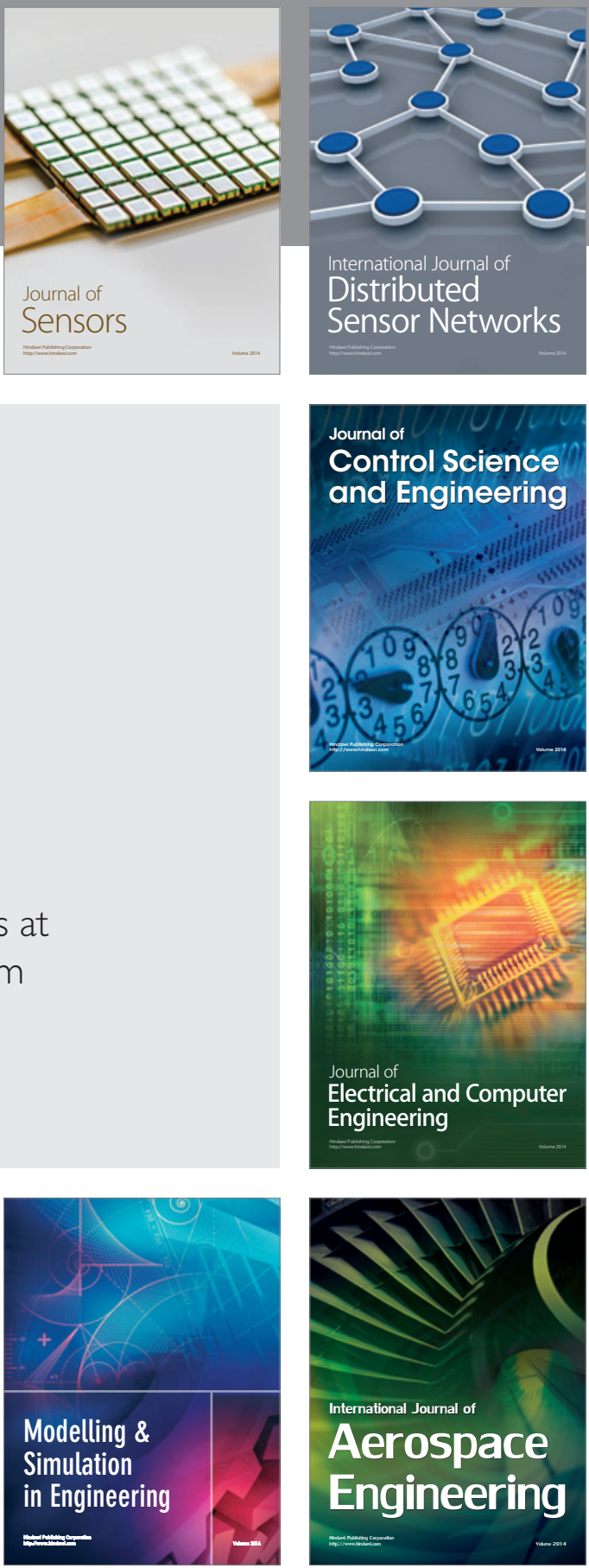

Journal of

Control Science

and Engineering
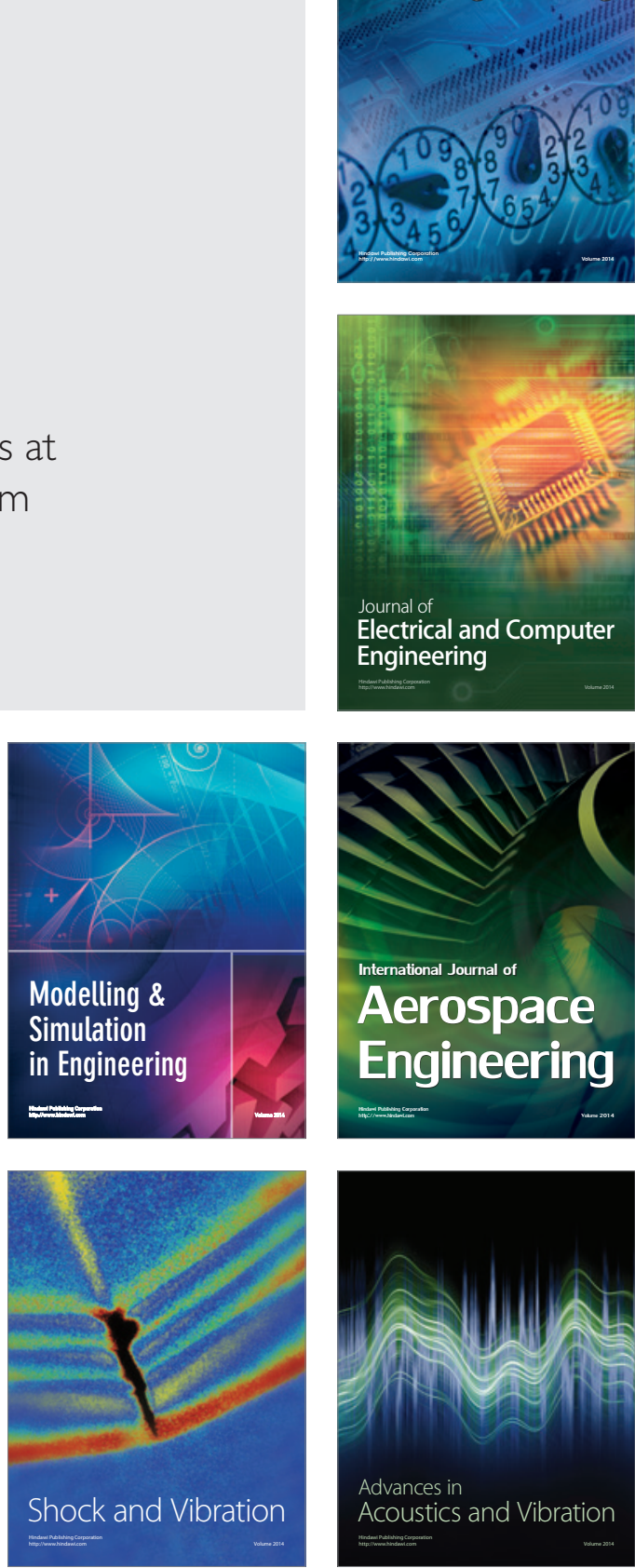